\title{
Optimal Cut-Off Value of the Superior Articular Process Area as a Morphological Parameter to Predict Lumbar Foraminal Stenosis
}

\author{
Tae-Ha Lim, ${ }^{1}$ Soo Il Choi, ${ }^{2}$ Hyung Rae Cho, ${ }^{3}$ Keum Nae Kang, ${ }^{4}$ Chang Joon Rhyu, ${ }^{4}$ \\ Eun Young Chae, ${ }^{5}$ Young Su Lim, ${ }^{2}$ Yongsoo Lee, ${ }^{1}$ and Young Uk Kim ${ }^{2}$ \\ ${ }^{1}$ Department of Anesthesiology and Pain Medicine, Eulji General Hospital, Eulji University College of Medicine, \\ Seoul, Republic of Korea \\ ${ }^{2}$ Department of Anesthesiology and Pain Medicine, Catholic Kwandong University of Korea College of Medicine, \\ International St. Mary's Hospital, Incheon, Republic of Korea \\ ${ }^{3}$ Department of Anesthesiology and Pain Medicine, Myongji Hospital, College of Medicine, Seonam University, \\ Goyang, Republic of Korea \\ ${ }^{4}$ Department of Anesthesiology and Pain Medicine, National Police Hospital, Seoul, Republic of Korea \\ ${ }^{5}$ Department of Radiology, Asan Medical Center, University of Ulsan College of Medicine, Seoul, Republic of Korea
}

Correspondence should be addressed to Young Uk Kim; zerg016@gmail.com

Received 27 June 2016; Accepted 21 November 2016; Published 9 January 2017

Academic Editor: Massimo Allegri

Copyright (C) 2017 Tae-Ha Lim et al. This is an open access article distributed under the Creative Commons Attribution License, which permits unrestricted use, distribution, and reproduction in any medium, provided the original work is properly cited.

Background. We devised a new morphological parameter called the superior articular process area (SAPA) to evaluate the connection between lumbar foraminal stenosis (LFS) and the superior articular process. Objective. We hypothesized that the SAPA is an important morphologic parameter in the diagnosis of LFS. Methods. All patients over 60 years of age were included. Data regarding the SAPA were collected from 137 patients with LFS. A total of 167 control subjects underwent lumbar magnetic resonance imaging (MRI) as part of a routine medical examination. We analyzed the cross-sectional area of the bone margin of the superior articular process at the level of L4-L5 facet joint in the axial plane. Results. The average SAPA was $96.3 \pm 13.6 \mathrm{~mm}^{2}$ in the control group and $128.1 \pm 17.2 \mathrm{~mm}^{2}$ in the LFS group. The LFS group was found to have significantly higher levels of SAPA $(p<0.001)$ in comparison to the control group. In the LFS group, the optimal cut-off value was $112.1 \mathrm{~mm}^{2}$, with $84.4 \%$ sensitivity, $83.9 \%$ specificity, and AUC of 0.94 (95\% CI: 0.91-0.96). Conclusions. Higher SAPA values were associated with a higher possibility of LFS. These results are important in the evaluation of patients with LFS.

\section{Introduction}

Lumbar spinal stenosis (LSS) results from degenerative changes in the spinal canal and is the most common spinal disease in elderly individuals [1-3]. It typically presents with buttock or low back pain, sensory and motor disturbances in the lower leg, and neurogenic intermittent claudication $[2,4]$. LSS is characterized by narrowing of the spinal canal, which is caused by disc herniation combined with osteophytes, hypertrophy of the ligamentum flavum, and mechanical compression of the spinal nerve roots $[5,6]$. Facet joint hypertrophy is considered another major cause of LSS [7]. Anatomically, degenerative LSS can involve the central canal, the foramina, the extraforaminal zone, or a combination of these locations. Lumbar foraminal stenosis (LFS) is defined as when the spinal nerve roots are compressed on the side due to narrowing of the foramen that may be caused by a foraminal herniated disc, a collapsed disc space, or an enlarged joint [6]. The foramen can be narrowed further by characteristic changes in the facet joints such as synovial cysts, osteoarthritis, or hypertrophy of articular processes $[8,9]$. All of the changes contribute to LFS symptoms $[4,5]$. Previous studies have indicated that morphologic parameters including the dural sac area, spinal canal area, and ligamentum flavum thickness 
are associated with disc degeneration, aging, and LSS [1012]. However, few studies have actually examined how facet joint hypertrophy affects LFS. The cross-sectional area of the superior articular process is an important morphologic parameter in the identification of facet joint hypertrophy [7]. Barry and Livesley measured the cross-sectional area of the superior articular process in the transverse plane using computed tomography [7]. However, they did not evaluate the role of SAPA as a morphological parameter of LFS. Therefore, in order to evaluate the connection between LFS and hypertrophy of the superior articular process, we devised a new morphological parameter called the superior articular process area (SAPA). The SAPA has not yet been evaluated for its association with LFS. We hypothesized that the SAPA is an important morphologic parameter in the diagnosis of LFS. Therefore, we used axial T2-weighted magnetic resonance imaging (MRI) to compare the SAPA between LFS patients and normal controls.

\section{Methods}

2.1. Patients. This study was registered at the Eulji University College of Medicine, Republic of Korea. The Institutional Review Board (IRB) reviewed and approved the research protocol. Patients who had visited the Spine Center between March 2014 and April 2016 and were diagnosed with LFS were reviewed retrospectively. Patients $>60$ years of age were included if they had clinical manifestations compatible with LFS (such as neurogenic intermittent claudication, sharp, dull, or radiating pain, numbness or weakness in the lower extremities, sensations of burning, and difficulty standing straight or walking), the most stenotic level occurred at L4-L5, and they had MRI imaging performed within 12 months of the diagnosis that was available for review. Patients were excluded if they had a past history of previous spinal injury or lumbar surgery, congenital spine defect, pain disorder, or history of spinal interventions such as kyphoplasty or vertebroplasty.

A total of 137 patients were enrolled after the LFS diagnosis was confirmed by an experienced, board-certified neuroradiologist. In LFS group, there were 66 (48.1\%) men and $71(51.8 \%)$ women with a mean age of $71.95 \pm 8.05$ years (range: 60-88 years) (Table 1). The NRS pain scores were recorded on an $11 \mathrm{~cm}$ horizontal line, with 0 indicating no pain and 10 indicating very severe pain. In order to compare the SAPA between patients with and without LFS, a group of control subjects was enrolled. The control patients had undergone lumbar MRI as part of a routine medical examination. Patients in the control group had no LSS-related symptoms. The control group consisted of 167 subjects ( 85 men and 82 women) with a mean age of $73.85 \pm 8.38$ years (range: 60-90 years) (Table 1). The SAPA in the control group was similarly examined at the L4-L5 facet joint level.

2.2. Imaging Parameters. MRI examinations were performed with 1.5 T (MAGNETOM Aera, MAGNETOM Espree, MAGNETOM Symphony, Sonata, Biograph, Avanto, Siemens Healthcare) and $3 \mathrm{~T}$ (MAGNETOM Skyra, MAGNETOM Verio, Siemens Healthcare) scanners. Axial and sagittal T2weighted images with $<3 \mathrm{~mm}$ slice thickness were obtained.
TABLE 1: Comparison of the characteristics of control and LFS groups.

\begin{tabular}{lcc}
\hline Parameter & $\begin{array}{c}\text { Control group } \\
(N=167)\end{array}$ & $\begin{array}{c}\text { LFS group } \\
(N=137)\end{array}$ \\
\hline Gender (male/female) & $85 / 82$ & $\begin{array}{c}66 / 71 \\
(\mathrm{NS})\end{array}$ \\
Age (years) & $73.85 \pm 8.38$ & $71.95 \pm 8.05$ \\
& & $(\mathrm{NS})$ \\
VAS score & & $5.9 \pm 1.1$ \\
SAPA $\left(\mathrm{mm}^{2}\right)$ & $96.31 \pm 13.60$ & $128.13 \pm 17.23$ \\
& $(p<0.001)$ \\
\hline
\end{tabular}

Data represent the mean \pm standard deviation (SD) or the numbers of patients.

LFS: lumbar foraminal stenosis; VAS: visual analog scale; SAPA: superior articular process area; NS: not statistically significant $(p>0.05)$.

The following other parameters were used: $0.9 \mathrm{~mm}$ intersection gap, $709 \mathrm{~ms} / 12 \mathrm{~ms}$ repetition time (TR)/echo time (TE), $30 \mathrm{~cm}$ field of view, $448 \times 314$ matrix, and $>3$ echo train length (ETL).

2.3. Image Analysis. Lumbar foraminal stenosis is defined as when the spinal nerve roots are compressed on the side due to narrowing of the foramen. The axial T2-weighted MR images were acquired at the level of facet joint for individual patient data. The INFINITT image analysis system (INFINITT; INFINITT Healthcare, Seoul, Korea) was used to measure the SAPA at the L4-L5 facet joint level on MRI. The SAPA was measured as the cross-sectional area by outlining the superior articular process at the narrowest foramen (Figure 1).

2.4. Statistical Analysis. The data are expressed as means \pm standard deviations (SD). Unpaired $t$-tests were used to compare the SAPA between the control and LFS groups. $p$ values $<0.05$ were considered statistically significant. The validity of the SAPA for diagnosis was estimated using receiver operating characteristic (ROC) curves, optimal cutoff values, area under the curve (AUC), sensitivity, and specificity with $95 \%$ confidence intervals (CIs). SPSS version 21 for Windows (IBM SPSS, IBM Corp., Armonk, NY) was used for the statistical analysis.

\section{Results}

A total of 167 subjects ( 85 men and 82 women) were included in the control group. The average age was $73.85 \pm 8.38$ years. And the average SAPA was $96.31 \pm 13.60 \mathrm{~mm}^{2}$ in the control group. A total of 137 patients (66 men and 71 women) were included in the LFS group. The average age and VAS score were $71.95 \pm 8.05$ years and $5.9 \pm 1.1$. The average SAPA was $128.13 \pm 17.23 \mathrm{~mm}^{2}$ in the LFS group. There were no significant differences between the groups with regard to age and sex. However, the LFS patients had significantly greater SAPA $(p<0.001)$ than did the control subjects (Table 1$)$. The ROC curve analysis showed that the optimal cut-off point of SAPA was $112.12 \mathrm{~mm}^{2}$ with $84.4 \%$ sensitivity, $83.9 \%$ specificity (Table 2), and AUC of 0.94 (95\% CI: 0.91-0.96) (Figure 2). 


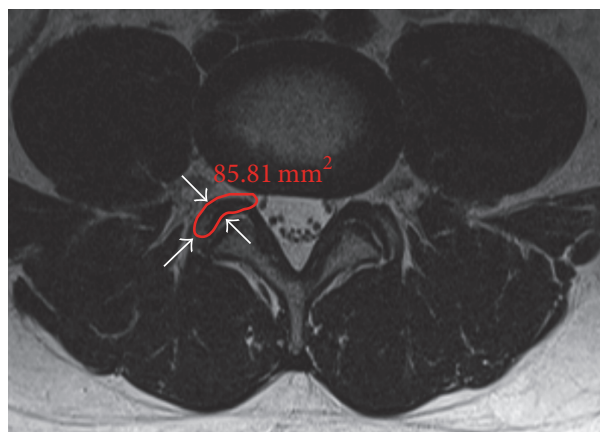

(a)

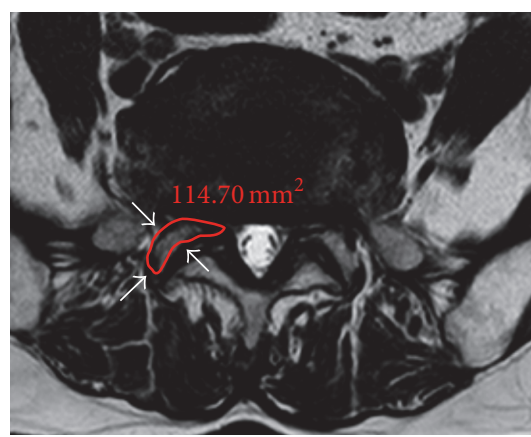

(b)

FIGURE 1: Measurement of the superior articular process area on MRI at the most stenotic level. (a) Control group. (b) Lumbar foraminal stenosis group.

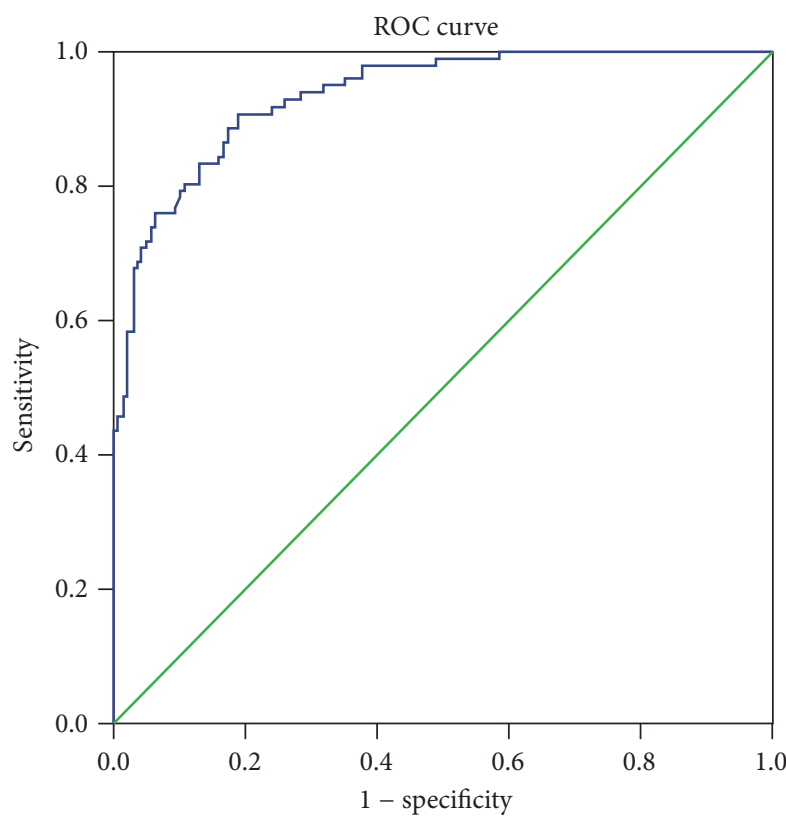

FIGURE 2: Receiver operating characteristic curve of superior articular process area for prediction of lumbar foraminal spinal stenosis. The best cut-off point of superior articular process area was $112.12 \mathrm{~mm}^{2}$, with sensitivity of $84.4 \%$, specificity of $83.9 \%$, and AUC of 0.94. AUC: area under the curve.

There were no statistically significant correlations between the NRS score and the SAPA.

\section{Discussion}

LSS is the most common spinal disorder in elderly patients that causes low back or buttock pain and intermittent neurogenic claudication $[2,13]$. LCSS results from a combination of pathogenic factors, including a decrease in the area of the cauda equina, hypertrophy of ligamentum flavum, loss of intervertebral disk height, and hypertrophy of the facet joints [14]. LFS results from posterolateral osteophytes from the vertebral endplates, which protrude into the foramen along with a herniated disk or a laterally bulging annulus fibrosus.
TABLE 2: Sensitivity and specificity of each cut-off point of the SAPA for prediction of lumbar foraminal spinal stenosis.

\begin{tabular}{lcc}
\hline SAPA $\left(\mathrm{mm}^{2}\right)$ & Sensitivity $(\%)$ & Specificity $(\%)$ \\
\hline 60.93 & 100 & 0 \\
99.82 & 95.8 & 62 \\
$112.12^{*}$ & 84.4 & 83.9 \\
114.63 & 65.5 & 89.8 \\
117.47 & 58.2 & 94.9 \\
170.74 & 0 & 100 \\
\hline
\end{tabular}

${ }^{*}$ The best cut-off point on the receiver operating characteristic curve. SAPA: superior articular process area.

Overgrowth of the facet joint capsule leads to foraminal stenosis $[6,15]$. Therefore, facet joint hypertrophy has been considered a major step in the development of LFS. Many previous studies have investigated the associations between the ligamentum flavum, dural sac area, spinal canal area on MRI, and the signs and symptoms of LSS. Park et al. reported that the ligamentum flavum is significantly thinner in patients with intervertebral disc herniation than it is in those with LSS [16]. Altinkaya et al. demonstrated that thickening of the ligamentum flavum was correlated to age, body mass index, and disc degeneration [17]. Ogikubo et al. found that there is a significant relationship between shorter walking distances and a smaller dural sac area [12]. Kim et al. reported that a larger dural sac area is associated with a longer subjective walking distance before the onset of claudication [11]. However, there are no previous reports of an association between LFS and facet joint hypertrophy as a morphologic parameter on MRI. Moreover, there are no objective morphologic parameters that indicate facet joint hypertrophy. Barry and Livesley reported that "facet joint hypertrophy" is a misnomer, because normal facet joints are no smaller than are degenerate facet joints [7]. This group also insisted that there is no clear definition in the literature regarding lumbar facet joint hypertrophy $[7,18]$. We believe that, in contrast to facet joint hypertrophy, the SAPA is the precise, objective measurement. Panjabi et al. described the cross-sectional area of the superior articular facet [19]. These cross-sectional areas were analyzed from autopsy specimens. Barry and Livesley 
measured the cross-sectional area of the superior articular process using computed tomography [7]. In this study, the SAPA was measured from MRI images. To our knowledge, this measurement has not been previously reported. Our results demonstrate associations between the SAPA and LFS. The LFS patients had significantly higher SAPA values than did control subjects. This study only included individuals $>60$ years of age because previous studies found that facet joints had only minimal cartilage changes before the age of 45 years and that osteoarthritic changes advance with age [8]. Our interpretation of these associations is that hypertrophy may be related to continuous stress, which might increase the SAPA. The process of facet joint hypertrophy begins with mechanical stress during lumbar rotation and flexion. These stressors put force on the facet joints, which leads to a high degree of abrasion [20,21]. This etiology may alter the morphologic features of the superior articular process. Bajek et al. explained that osteophyte formation in the lumbar spine is an attempt to stabilize an unstable segment; this mechanism ultimately leads to facet hypertrophy [22]. AlRawahi et al. have demonstrated that mechanical influences tended to increase with the size of osteophyte. Osteophytes contribute $7 \%$ to $9 \%$ of the bone mineral density measurement for a motion segment [23]. Disc degeneration may also increase the stressful force on the facet joints [24]. Therefore, osteophytes and hypertrophy of the superior articular process were the main factors of bone tissue [25]. Our results indicate that the visual analog scale score is not significantly associated with the SAPA on MRI. We believe that the pathophysiology of spinal stenosis is multifactorial [26]. Although mechanical compression of the spinal canal is a major factor, subjective discomfort, inflammatory effects [27-29], and depression may also contribute to the disease's pain intensity [30].

This study has several limitations. First, there may be errors associated with measuring the SAPA on MRI. We measured the SAPA in the axial T2 images at the level of the L4-L5 facet joint. However, these axial images may be heterogeneous due to differences in the cutting angle of the MRI resulting from individual anatomic variations and technical problems. A $3.0 \mathrm{~mm}$ slice of axial T2-weighted MR image is also thicker than an ideal slice. Second, we did not measure the sagittal cut to calculate the surface area of the superior articular process. Third, degenerative LSS can involve the central canal, the foramina, the extraforaminal zone, or a combination of these locations. However, we focused on LFS. Our analysis would be improved if data including the SAPA of the LCSS and extraforaminal entrapment were compared with our current findings. Fourth, there are several different methods that are known to be effective at discriminating LSS, such as sedimentation sign or morphologic grading $[6,31]$. However, this study only used SAPA measurement. Therefore, our results may be limited regarding measurement of the epidural pressure or morphologic changes. Fifth, we excluded the inferior articular process area because this area blurs the boundaries. It is difficult to accurately measure the inferior articular process area in each level due to the overlap. Finally, another limitation of this study is its retrospective nature.

Despite these limitations, this is the first study to document that the SAPA is associated with LFS. These results may help treating physicians recognize an important cause of LFS.

\section{Conclusions}

Our results demonstrate that SAPA is a sensitive measurement parameter for assessing LFS. With regard to LFS, the optimal cut-off value was $112.12 \mathrm{~mm}^{2}$, with $84.4 \%$ sensitivity, 83.9\% specificity, and AUC of 0.94 . We believe that this result will help physicians in their evaluations of patients with LFS.

\section{Competing Interests}

The authors declare that they have no competing interests.

\section{Authors' Contributions}

Tae-Ha Lim, Soo Il Choi, and Young Uk Kim helped design the study, conduct the study, analyze the data, and write the manuscript. Hyung Rae Cho helped design the study. Keum Nae Kang helped conduct the study and analyze the data. Chang Joon Rhyu, Eun Young Chae, and Young Su Lim helped conduct the study. Yongsoo Lee revised the manuscript. Tae-Ha Lim and Soo Il Choi have seen the original study data, reviewed the analysis of the data, approved the final manuscript, and are the authors responsible for archiving the study files. Hyung Rae Cho, Keum Nae Kang, Chang Joon Rhyu, Eun Young Chae, Young Su Lim, and Young Uk Kim have seen the original study data, reviewed the analysis of the data, and approved the final manuscript. Yongsoo Lee reviewed the analysis of the data. Tae-Ha Lim and Soo Il Choi equally contributed to this paper as co-first authors.

\section{References}

[1] S. Costandi, B. Chopko, M. Mekhail, T. Dews, and N. Mekhail, "Lumbar spinal stenosis: therapeutic options review," Pain Practice, vol. 15, no. 1, pp. 68-81, 2015.

[2] A. J. Haig and C. C. Tomkins, "Diagnosis and management of lumbar spinal stenosis," JAMA, vol. 303, no. 1, pp. 71-72, 2010.

[3] L. Kalichman, R. Cole, D. H. Kim et al., "Spinal stenosis prevalence and association with symptoms: the Framingham Study," Spine Journal, vol. 9, no. 7, pp. 545-550, 2009.

[4] J. H. Hong, M. Y. Lee, S. W. Jung, and S. Y. Lee, "Does spinal stenosis correlate with MRI findings and pain, psychologic factor and quality of life?" Korean Journal of Anesthesiology, vol. 68, no. 5, pp. 481-487, 2015.

[5] A. Yuz, S. Makirov, and V. Osadchiy, "Measuring spinal canal size in lumbar spinal stenosis: description of method and preliminary results," International Journal of Spine Surgery, vol. 9, article no. 3, 2015.

[6] T. Ohba, S. Ebata, K. Fujita, H. Sato, C. J. Devin, and H. Haro, "Characterization of symptomatic lumbar foraminal stenosis by conventional imaging," European Spine Journal, vol. 24, no. 10, pp. 2269-2275, 2015.

[7] M. Barry and P. Livesley, "Facet joint hypertrophy: the crosssectional area of the superior articular process of L4 and L5," European Spine Journal, vol. 6, no. 2, pp. 121-124, 1997.

[8] S. Ko, A. R. Vaccaro, S. Lee, J. Lee, and H. Chang, "The prevalence of lumbar spine facet joint osteoarthritis and its 
association with low back pain in selected Korean populations," Clinics in Orthopedic Surgery, vol. 6, no. 4, pp. 385-391, 2014.

[9] H. S. Jin, J. Y. Bae, C. B. In, E. J. Choi, P. B. Lee, and F. S. Nahm, "Epiduroscopic removal of a lumbar facet joint cyst," Korean Journal of Pain, vol. 28, no. 4, pp. 275-279, 2015.

[10] J. Abbas, K. Hamoud, Y. M. Masharawi et al., "Ligamentum flavum thickness in normal and stenotic lumbar spines," Spine, vol. 35, no. 12, pp. 1225-1230, 2010.

[11] Y. U. Kim, Y.-G. Kong, J. Lee et al., "Clinical symptoms of lumbar spinal stenosis associated with morphological parameters on magnetic resonance images," European Spine Journal, vol. 24, no. 10, pp. 2236-2243, 2015.

[12] O. Ogikubo, L. Forsberg, and T. Hansson, "The relationship between the cross-sectional area of the cauda equina and the preoperative symptoms in central lumbar spinal stenosis," Spine, vol. 32, no. 13, pp. 1423-1428, 2007.

[13] S. Genevay and S. J. Atlas, "Lumbar spinal stenosis," Best Practice and Research: Clinical Rheumatology, vol. 24, no. 2, pp. 253-265, 2010.

[14] H. J. Park, S. S. Kim, Y. J. Lee et al., "Clinical correlation of a new practical MRI method for assessing central lumbar spinal stenosis," The British Journal of Radiology, vol. 86, no. 1025, Article ID 20120180, 2013.

[15] M. Yoshimoto, T. Takebayashi, S. Kawaguchi et al., "Minimally invasive technique for decompression of lumbar foraminal stenosis using a spinal microendoscope: technical note," Minimally Invasive Neurosurgery, vol. 54, no. 3, pp. 142-146, 2011.

[16] J. B. Park, H. Chang, and J. K. Lee, "Quantitative analysis of transforming growth factor- $\beta 1$ in ligamentum flavum of lumbar spinal stenosis and disc herniation," Spine, vol. 26, no. 21, pp. E492-E495, 2001.

[17] N. Altinkaya, T. Yildirim, S. Demir, O. Alkan, and F. B. Sarica, "Factors associated with the thickness of the ligamentum flavum: is ligamentum flavum thickening due to hypertrophy or buckling?" Spine, vol. 36, no. 16, pp. E1093-E1097, 2011.

[18] L. J. Grobler, P. A. Robertson, J. E. Novotny, and M. H. Pope, "Etiology of spondylolisthesis: assessment of the role played by lumbar facet joint morphology," Spine, vol. 18, no. 1, pp. 80-91, 1993.

[19] M. M. Panjabi, T. Oxland, K. Takata, V. Goel, J. Duranceau, and M. Krag, "Articular facets of the human spine: quantitative three-dimensional anatomy," Spine, vol. 18, no. 10, pp. 1298-1310, 1993.

[20] R. B. Dunlop, M. A. Adams, and W. C. Hutton, "Disc space narrowing and the lumbar facet joints," Journal of Bone and Joint Surgery B, vol. 66, no. 5, pp. 706-710, 1984.

[21] J. Wang and X. Yang, "Age-related changes in the orientation of lumbar facet joints," Spine, vol. 34, no. 17, pp. E596-E598, 2009.

[22] G. Bajek, S. Bajek, S. Z. Cvek, D. Bobinac, B. Splavski, and D. S. Grahovac, "Histomorphological analysis of the osteophytic appositions in patients with lumbar lateral recess syndrome," Collegium Antropologicum, vol. 34, no. 2, pp. 79-84, 2010.

[23] M. Al-Rawahi, J. Luo, P. Pollintine, P. Dolan, and M. A. Adams, "Mechanical function of vertebral body osteophytes, as revealed by experiments on cadaveric spines," Spine, vol. 36, no. 10, pp. 770-777, 2011.

[24] C. D. Chaput, J. J. Allred, J. J. Pandorf, J. Song, and M. D. Rahm, "The significance of facet joint cross-sectional area on magnetic resonance imaging in relationship to cervical degenerative spondylolisthesis," Spine Journal, vol. 13, no. 8, pp. 856-861, 2013.
[25] H. Zhou, G. Dong, H. Huang, Z.-M. Xia, and Z.-H. Zhang, "MRI finding of the lumbar foraminal stenosis and its clinical significance," China Journal of Orthopaedics and Traumatology, vol. 23, no. 8, pp. 587-590, 2010.

[26] C. H. Park, S. H. Lee, and J. Y. Jung, "Dural sac cross-sectional area does not correlate with efficacy of percutaneous adhesiolysis in single level lumbar spinal stenosis," Pain Physician, vol. 14, no. 4, pp. 377-382, 2011.

[27] S. H. Park, P. B. Lee, G. Y. Choe, J. Y. Moon, F. S. Nahm, and Y. C. Kim, "Therapeutic effect of epidurally administered lipo-prostaglandin E1 agonist in a rat spinal stenosis model," Korean Journal of Pain, vol. 27, no. 3, pp. 219-228, 2014.

[28] S. S. Choi, E. Y. Joo, B. S. Hwang et al., "A novel ballooninflatable catheter for percutaneous epidural adhesiolysis and decompression," Korean Journal of Pain, vol. 27, no. 2, pp. 178$185,2014$.

[29] J. M. Byun, H. S. Park, J. H. Woo, and J. Kim, “The effects of a forceful transforaminal epidural steroid injection on radicular pain: a preliminary study," Korean Journal of Pain, vol. 27, no. 4, pp. 334-338, 2014.

[30] T. Giesecke, R. H. Gracely, D. A. Williams, M. E. Geisser, F. W. Petzke, and D. J. Clauw, "The relationship between depression, clinical pain, and experimental pain in a chronic pain cohort," Arthritis and Rheumatism, vol. 52, no. 5, pp. 1577-1584, 2005.

[31] T. Barz, M. Melloh, L. P. Staub, S. J. Lord, J. Lange, and H. R. Merk, "Increased intraoperative epidural pressure in lumbar spinal stenosis patients with a positive nerve root sedimentation sign," European Spine Journal, vol. 23, no. 5, pp. 985-990, 2014. 


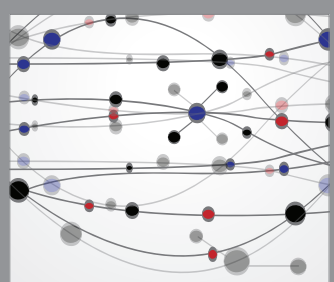

The Scientific World Journal
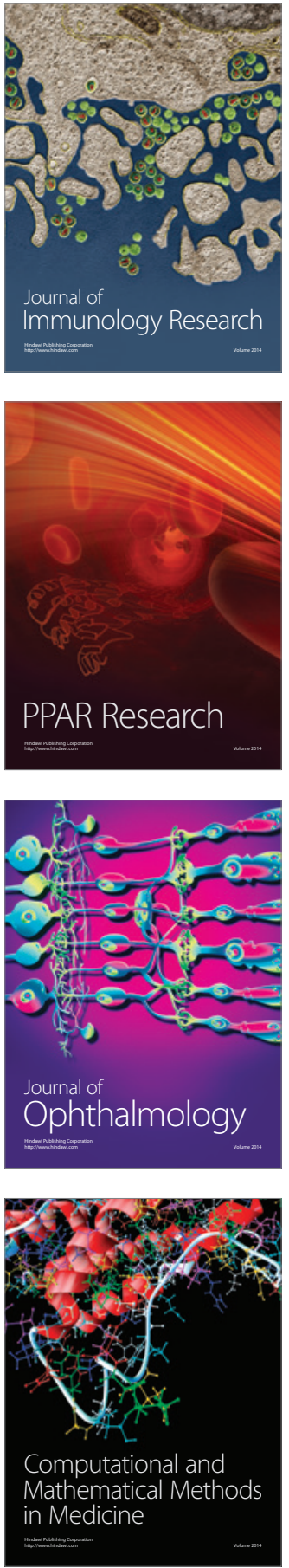

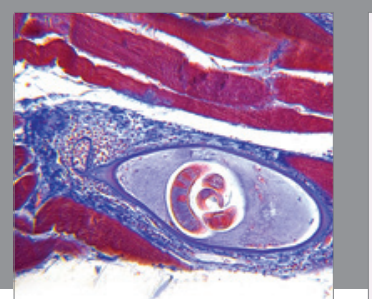

Gastroenterology Research and Practice
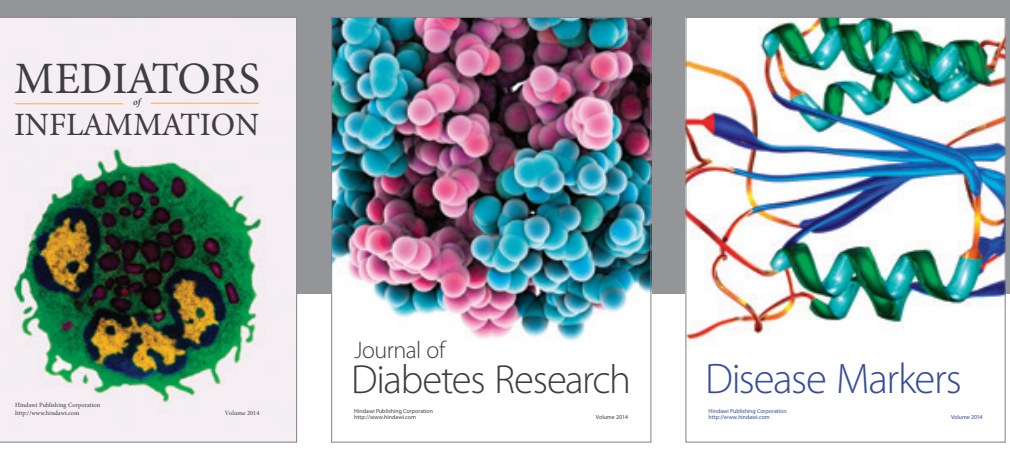

Disease Markers

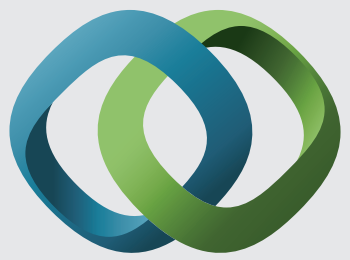

\section{Hindawi}

Submit your manuscripts at

https://www.hindawi.com
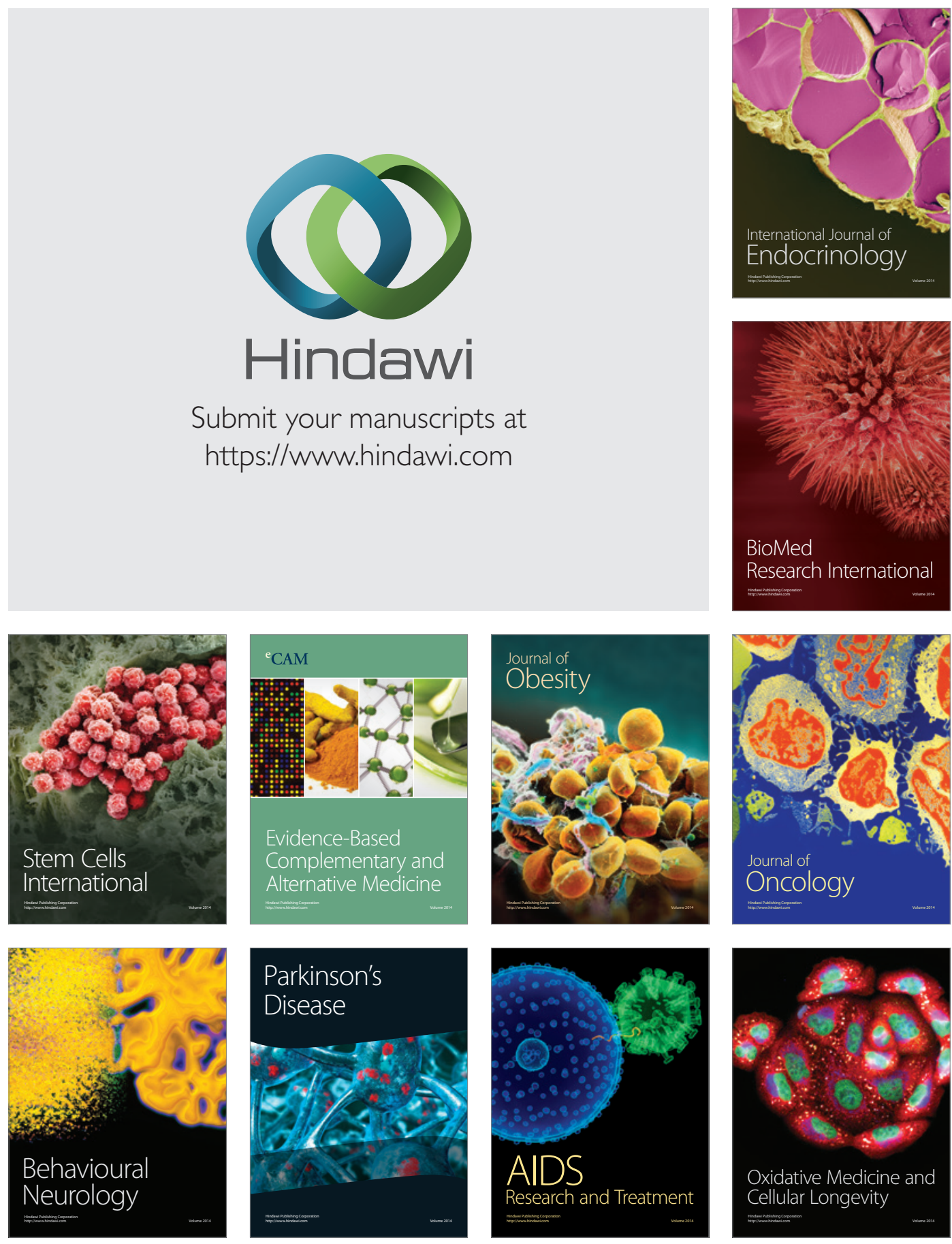